\title{
Evaluation of trauma patterns in blast injuries using multiple correspondence analysis
}

Marie Christine Dussaulta, $b,{ }^{*}$, Martin Smithb, Ian Hansonc

a University of Pretoria, Department of Anatomy, Faculty of Health Sciences, Basic Medical Sciences, Room 5-3, 9 Bophelo Way, Pretoria 0001, South Africa

b Bournemouth University, Faculty of Science and Technology, Bournemouth, Fern Barrow, Poole, Dorset BH12 5BB, United Kingdom

c International Commission on Missing Persons, Alipašina 45A, Sarajevo 71000, Bosnia and Herzegovina

Keywords: Trauma, Anthropology,Blast, Gunshot, Multiple correspondence analysis

\section{A B S T R A C T}

Anthropology features little in published literature about blast injuries. Contributions through case studies and experimental research are beginning to expand our understanding of the effect these injuries have on the human skeleton. This study examines blast injury and gunshot related fractures through multiple correspondence analysis (MCA) with the aim of establishing injury patterns between the two types of trauma. Using a sample of 491 individuals from Bosnia, MCA is employed to identify which body regions differentiate between blast or gunshot related fractures. Cranial fractures were more closely associated with gunshot related cases. Post-cranial fractures were associated with blast-related cases. A differentiation in post-cranial and cranial fractures between gunshot and blast related cases was revealed in the samples. The high prevalence of extremity trauma in blast is similar to previous work, but the smaller amount of cranial blast-related fractures differs from previous studies and from what is found in gunshot-related cases. Differentiation of blast and gunshot wound injuries can be made on the human skeleton and can be used to possibly interpret injury mechanism in large skeletal assemblages as well as single cases.

\section{Introduction}

Clinical publications are the foundation for knowledge on blast injuries in the human skeleton. In the last ten years, research on blast injuries has primarily targeted the pathogenesis and etiology of traumatic brain injury along with the medical management of musculoskeletal injuries and limb salvage [1-5]. In the anthropological analysis of trauma, examination of blast injury features little in published literature, with most information associated with case studies [6,7] and some experimental research [8]. Medical classification of blast injuries is a four-tiered system that was developed on the basis of blast physics and mechanism of injury $[9,10]$. Primary blast injury is the result of blast wave overpressure and affects the air or fluid filled organs. Experimental work links primary blast injury with skeletal trauma to the ribs and traumatic amputations $[8,11,12]$. Secondary blast injury represents injuries caused by the fragmentation of munitions and the impact of these fragments on the human body. This includes fractures, often represented by irregular fracturing of bone with character- istics of ballistic injury [13]. Tertiary blast injury results from the movement and impact of the body against an immovable object. 
Injuries from the impact of objects into the body are also classified as tertiary. Tertiary injuries can result in blunt force fractures to skeletal elements. Quaternary blast injuries refer to thermal and chemical damage to the body [9]. As a blast is a broad-focused force, injuries are predominantly distributed throughout the body [14]. However, the location of the explosion to a victim does influence fracture variation. Other variables, such as whether the person is an open or enclosed environment can also influence the pattern of trauma [14-17]. For example, terrorist bombing events (occurring in both enclosed and open areas) typically result in a high prevalence of extremity injuries, particularly to the head and lower limbs [1820].

Contrasting this pattern, suicide bombings have a greater number of soft tissue injuries rather than fractures. This may be due to the inclusion of shrapnel-type materials from improvised sources such as ball bearings, pieces of metal and nails [7]. In general, fractures occur typically in the lower limbs in both terrorism and suicide bombings [14,21-24]. However if both types of bombings occur in a confined area (such as a building), the blast overpressure will be reflected back onto the victims and will result in a potentially higher number of primary blast injuries [25,26]. Patterns of injury in gunshot wounds vary from those of blast injuries, and an understanding of the observable fractures patterns is useful for differentiating them [10]. Common areas affected by gunshot wounds are the chest, spine and abdomen and include injuries concurrently seen in only one or two body regions, whereas blast injuries, as mentioned above, are more diffuse across the skeleton [27,28]. Forensic contexts often address questions regarding the distribution and nature of blastrelated fractures and their similarities or differences to gunshot related injuries. Both the identification and differentiation of blast and gunshot related fractures has been legally questioned [29] in cases of human rights abuses. To date, baseline data are not available from which to compare blast and gunshot related injuries and to assess whether the certain trauma patterns are consistent with these types of trauma. Therefore, the purpose of this study is to describe blast fracture patterns and to compare, and possibly differentiate, from the gunshot injury fracture patterns in the human skeleton.

\section{Materials and methods}

The International Commission on Missing Persons in Sarajevo, Bosnia (ICMP) provided pathology reports, anthropology reports, autopsy photographs and site photographs from the analysis of five mass graves from the Glogova, Zeleni Jadar and Lazete excavations. The total sample size was 491 males, aged from 8 to 75 years. Pathologists provided the cause and manner of death of each person based on observed trauma (in both soft and hard tissues) and associated evidence, such as shrapnel embedded in the tissues. Cause of death was determined to be either gunshot wound or blast. Forensic investigators used physical evidence to corroborate the cause and manner of death and demonstrated associated use of rocket-propelled grenades for the blast-related cases [30], also supported by witness statements. Only cases in which a cause of death was determined were included in the sample. Cases with blast related fractures were from an enclosed context. The primary author assessed whether the damage was perimortem or postmortem before comparing the bone injuries to the soft tissue trauma in the pathology reports. This confirmed inclusion in the study based on presence of perimortem blast or gunshot-related fractures, for each individual case. Perimortem damage identification followed guidelines from previously published studies commonly employed in forensic anthropology practice [31-33], and is the differentiation of fractures on wet and dry bone [34,35]. 
The distinction between perimortem and postmortem fractures used characteristics of the angle, outline and edge of the fractures [36]. Perimortem fractures characteristics were an oblique (eitherobtuse or acute) angle between the fracture surface and bone cortical surface, three types of fracture outline (transverse, curved or v-shaped) and the texture of the fracture margin (smooth, straight margins associated with wet bone fractures). Postmortem characteristics were a right fracture angle, jagged edges to the texture of the fracture as well as colorvariation of the fracture surface and the internal and external bone surfaces. Cases with extensive post-mortem damage and cases with ambiguous distinctions between fresh and dry bone fractures were excluded from the study. With selected perimortem fracture cases, the type of trauma was assessed. Forensic pathologists identified morphological characteristics of gunshot wounds such as entrance and exit defects (with or without associated bullet fragments), bullet tracks, fragmentation patterns in bone and the presence of intact or fragmented bullets in the soft tissues. Shrapnel trauma and blast injury was identified by the presence of irregular damage in bone which is not typical of gunshot or blunt force trauma and was associated with shrapnel and sometimes charring [37].

Data compiled from the reports were entered into a Microsoft ExcelTM spreadsheet. A dichotomous scoring system (represented by 1 for absent and 2 for present) was used to identify which body regions had perimortem fractures, as detailed in Table 1 . The divisions were created so as to specifically focus on an individual bone, or groups of closely related bones in one body region. Each case was classified by type of trauma, either gunshot or blast related fractures. Multiple correspondence analysis (MCA) was used within SPSS (19.0). MCA was used to examine sample variance as a measure of similarity or dissimilarity between the perimortem, or wet bone, fracture patterns among the various body regions. The greater the variance between two samples in the study the more dissimilar they are. By selecting the variables that account for the most variance and graphically projecting these onto three axes, observable differences between the patterns of fractures can be visualised.

The sample of cases with gunshot related fractures was much larger than the sample with blast related fractures. Multiple correspondence analysis performs better when using approximately equal sample sizes. To ensure sample size did not influence analysis, random sub-sampling of the gunshot related fracture sample was performed. The primary author used a Mersene Twister random number generator to select cases (approximately 80 individuals each) from the total population. Employing a sub-sampled group makes use of the total sample as a population and reduces the effect of confounding variables due to unequal sample sizes. To analyse the variance in the patterns between two types of trauma, point clouds were produced. Each point representing one individual and is labelled by the type of trauma, either blast or gunshot-related fractures. This method permits the visualisation of clusters and patterns within the sample. Plotting the variables (body regions) against each reduced dimension identifies which body regions are associated with two types of trauma (labelled as gunshot and blast) and this is used to differentiate the groups. Each analysis produces a model summary, which includes the percentage of variance accounted for by each dimension.

The ICMP provided ethical approval to use the samples, along with additional approval from the ethics committee at Bournemouth University. To address anonymity of the data, the authors used no identifying features (including discussion of individual cases and case numbers). Each case used the ICMP assigned case numbers for identification during analysis only. 


\section{Results}

Examining the overall frequencies, the gunshot wound group has bone trauma concentrated in the neurocranium, vertebral column and ribs. The blast injury group also has injury in these areas but with equal amounts of trauma to other regions such as the upper and lower limbs. With MCA, the analysis reduces the data to three dimensions that account for $33 \%$ of the variance in the samples. The variables of Dimension 1 have the most inertia $(0.130)$ and contribute most of the differences between the two types of trauma-related fractures. Fig. 1 shows the frequency of blast or gunshot-related fractures for each body region. The differentiation in two types of injuries are shown in Fig. 2 for all cases. On Dimension 1 gunshot-related fractures cluster on the positive side of the axis and blast-related fractures on the negative side of the axis. In Fig. 3, blast-related fractures cluster on the positive side; and gunshot-related fractures cluster on the negative side of Dimension 3, while retaining the same pattern for Dimension 1.

To determine which body regions contribute the most to the two types of trauma-related fracture patterns, joint plots of category points were produced (Figs. 4 and 5). Dimension 1 is the distribution cranial and postcranial trauma in the samples and Dimension 2 is the presence and absence of trauma. The plot of category points (variables) shows clustering of blast related trauma in the post-cranial body regions, on the negative side of Dimension 1 , and gunshot-related trauma clusters with the mandible, maxillofacial and neurocranium, on the positive side of the axis.

The second dimension highlights the spread of fractures in the body. Blast-related injuries have a diffuse pattern of fractures throughout the body, whereas gunshot-related fractures are more focused to particular regions. In the joint plot of category points for Dimension 1 and Dimension 3, gunshot and blast related trauma clusters on opposite sides of the axis. Dimension 3 re-iterates the results of Dimension 2, a difference in the spread of fractures in blast and gunshot cases.

\section{Discussion/conclusion}

Fracture patterns varied between the two samples and is consistent with the current literature on both types of trauma $[10,14,18-20,27,28]$. Gunshot injuries cluster in the cranial regions with blast-related injuries in the postcrania. Blast-related cases spread more diffusely spread throughout the body than gunshot related cases. Gunshot and blast related injuries differ in cranial and postcranial element distinction as well as present a focused versus diffuse fracture pattern throughout the body. The results in this study concur with previously published studies that examine various contexts of blast injury [14,18-20]. Variations in the observed patterns, with other literature on blast injuries, may be related to a difference in the context (such as combat versus civilian cases), geographical differences (open air or enclosed) as well the type of explosive material and its construction (such as improvised explosive devices linked with terrorism).

Combat-related blast injuries typically occur in all body regions, and often exclude the head [38]. This has been attributed to the dispersive methods of the modern explosive materials that reflect upon a larger surface area of the body, particularly the post-cranial region $[53,54]$. Open-air and enclosed blast situations are similar with the latter having the added implications of blast wave reflection and building collapse. This may be of relevance to the 
interpretation of injuries from this study as those with blast related fractures were from an enclosed context. In open-air related terrorism blasts, injuries are usually found in the head, neck and periphery $[18,39]$ and are often associated with secondary and tertiary blast injuries. Multiple areas of injury are usually described as a diffuse pattern, with the majority of injuries occurring in the extremities, especially the distal portion of the limbs [13,27,4042], as well as facial and skull fractures [44,45,47]. This aspect of open-air blast is consistent with observations in the sample as well as with other contexts such as enclosed blast. Enclosed blast injuries present with similar diffuse and broad patterns of injuries but with more fragmentation caused from secondary blast trauma, or shock waves. In the Oklahoma City bombing, $75 \%$ of the victims had extremity trauma [43]; whereas in the Madrid train bombings, the head and neck area was the most injured followed with the extremities $[28,48,49]$. The diffuse injury and fragmentation of the extremities in the Bosnian blast-injury cases were associated with a rocket-propelled grenade that had been launched into a closed environment; therefore the fracture patterns are expected to be more similar to an enclosed contexts rather than an open air context [45].

The diffuse fracture patterns in blast-injuries may be difficult to distinguish between open-air and enclosed sites, but the fracture patterns are distinct, and less localised, than those observed with gunshot injuries, and therefore can be distinguished from them. Gunshotrelated trauma is associated with more specific targeting of certain regions for the production of maximum damage [46]. This presentation actually differs from that seen in other studies of terrorist gunshot related trauma patterns, which show that the chest, spine and abdominal areas are affected $[27,28]$. A recent study of human rights cases of gunshot related trauma showed that injuries to the head and torso predominate [46]. This differs from our study showing predominance of gunshot wound related fractures in the head area, but not in the torso. The authors also examined the distribution of injuries in the body, noting that discrete areas were targeted and suggests the intention of killing rather than injuring the victims. This is also seen in our study with the pattern of gunshot wound related fractures being concentrated in the cranial rather than the postcranial skeletal elements. Blast injuries, while associated with ballistic trauma, presents with characteristic morphology of blunt injuries, this is likely on account of the diffuse blast wave transferring energy over a larger area and the energy loading being slower than a gunshot at the point of impact. For example, Christensen et al. [8] conducted experimental research on the effect of open-aired blast-injuries showing diffuse and extensively comminuted fractures throughout the body. They classified the fracture pattern observed as more random than is typically associated with gunshot and/or blunt force injuries. However, the pattern of blast related fractures observed in our study resembles patterns of blunt force trauma on account of the diffuse energy of the blast wave over a larger surface area. Large area transfer of energy in falls from heights also presents with similar fracture patterns as those seen on blast related injuries [47].

Blast-related fractures are complex in their identification. However, some important patterns observed in both the published materials and the known blast-related fracture cases from Bosnia can guide anthropological analysis of this type of trauma and its differentiation from gunshot related fractures on an assemblage basis. Particularly, a cranial and postcranial distinction in the presentation of fractures is consistent with differing the type of trauma, with the blast-related fractures occurring typically postcranially and often in the extremities. Observations of this distinction in a total body trauma pattern can contribute to possible identification of blast trauma in cases where it is suspected and corroborated by other 
evidence, such as the presence of physical evidence. Diffuse distribution of fractures is also more similar to the patterns of fractures in blunt force trauma and may resemble cases such as falls from heights rather than isolate gunshot wounds. These conclusions concur with previous studies of terrorist blast incidents, combat blast related trauma and experimental studies and aid in guiding physical anthropologists in the analysis of fractures related to blast in forensic contexts. This is relevant to organisations such as the ICMP and the International Criminal Tribunal for the Former Yugoslavia who continue investigations on interred victims and require new approaches to case analysis. Examination of patterns of injury can contribute to the corroboration of documented events and witness statements for the identification of groups of related victims as well as informing the extent of archaeological search procedures and re-association of elements from mass graves in cases of suspected blast-related fractures by contributing information on the expected pattern of fracturing in such cases.

\section{Acknowledgements}

The authors wish to thank the International Commission on Missing Persons for access to the data. Many thanks to EN L'Abbé for comments and edits on earlier drafts. This research did not receive any specific grant from funding agencies in the public, commercial, or not-forprofit sectors.

\section{References}

[1] N. de Lanerolle, J. Kim, F. Bandak, Neuropathology of traumatic brain injury: comparison of penetrating, nonpenetrating direct impact and explosive blast etiologies, Semin. Neurol. 35 (2015) 012-019.

[2] G.A. Elder, A. Cristian, Blast-related mild traumatic brain injury: mechanisms of injury and impact on clinical care, Mt. Sinai J. Med.: J. Transl. Personalized Med. 76 (2009) 111-118.

[3] P.D. Katherine, H. Taber, M.D. Deborah, L. Warden, M.D. Robin, A. Hurley, Blastrelated traumatic brain injury: what is known? J. Neuropsychiatry Clin.

Neurosci. 18 (2) (2006) 141-145.

[4] A.B. Richard, L. Geoffrey, T. Lawrence, J. Adolph, A. Denes, D. Nihal, et al., An introductory characterization of a combat-casualty-care relevant swine model of closed head injury resulting from exposure to explosive blast, J. Neurotrauma 26 (2009) 841-860.

[5] A.I. Schneiderman, E.R. Braver, H.K. Kang, Understanding sequelae of injury mechanisms and mild traumatic brain injury incurred during the conflicts in Iraq and Afghanistan: persistent postconcussive symptoms and posttraumatic stress disorder, Am. J. Epidemiol. 167 (2008) 1446-1452.

[6] M.T. Allaire, M.H. Manhein, Suicide by blasting caps: a case study of rare cranial trauma, J. Forensic Sci. 53 (2008) 1313-1315 (Blackwell Publishing Limited).

[7] E. Kimmerle, J.P. Baraybar, Skeletal Trauma: Identification of Injuries Resulting from Human Rights Abuse and Armed Conflict, CRC Press, London, 2008.

[8] A.M. Christensen, V.A. Smith, V. Ramos, C. Shegogue, M. Whitworth, Primary and secondary skeletal blast trauma, J. Forensic Sci. 57 (2012) 6-11.

[9] D.S. Plurad, Blast injury, Mil. Med. 176 (2011) 276-282.

[10] S.A. Stapley, L.B. Cannon, (i) An overview of the pathophysiology of gunshotand blast injury with resuscitation guidelines, Curr. Orthop. 20 (2006) 322-332.

[11] A.M. Christensen, V.A. Smith, Rib butterfly fractures as a possible indicator of blast trauma, J. Forensic Sci. 58 (2013) S15-S19. 
[12] J.B. Hull, G. Cooper, Pattern and mechanism of traumatic amputation by explosive blast, J. Trauma Inj. Infect. Crit. Care 40 (1996) 198S-205S.

[13] Y.A. Weil, K. Petrov, M. Liebergall, Y. Mintz, R. Mosheiff, Long bone fractures caused by penetrating injuries in terrorists attacks, J. Trauma 62 (2007)

909-912.

[14] D.D. Ad-El, A. Eldad, Y. Mintz, Y. Berlatzky, A. Elami, A.I. Rivkind, et al., Suicide bombing injuries: the Jerusalem experience of exceptional tissue damage posing a new challenge for the reconstructive surgeon, J. Plast. Reconstr. Surg. 118 (2006) 383-387.

[15] E. Chaloner, Blast injury in enclosed spaces, Br. Med. J. 331 (2005) 119-120.

[16] A. Ramasamy, S.D. Masouros, N. Newell, A.M. Hill, W.G. Proud, K.A. Brown, et al., In-vehicle extremity injuries from improvised explosive devices: current and future foci, Philos. Trans. R. Soc. B: Biol. Sci. 366 (2011) 160-170.

[17] Y. Kluger, Bomb explosions in acts of terrorism - detonation, wound ballistics triage and medical concerns, Isr. Med. Assoc. J. 5 (2003) 235-240.

[18] W.A. Hadden, W.H. Rutherford, J.D. Merrett, The injuries of terrorist bombing: a study of 1532 consecutive patients, Br. J. Surg. 65 (1978) 525-531.

[19] D.P. Rignault, M.C. Deligny, The 1986 terrorist bombing experience in Paris, Ann. Surg. 209 (1989) 368-373.

[20] M. Martí, M. Parrón, F. Baudraxler, A. Royo, N.G. León, R. Álvarez-Sala, Blast injuries from Madrid terrorist bombing attacks on March 11, 2004, Emerg.

Radiol. 13 (2006) 113-122.

[21] L. Aharonson-Daniel, Y. Klein, K. Peleg, Suicide bombers form a new injury profile, Ann. Surg. 244 (2006) 1018-1023.

[22] G. Almogy, H. Belzberg, Y. Mintz, A.K. Pikarsky, G. Zamir, A.I. Rivkind, Suicide bombing attacks: update and modifications to the protocol, Ann. Surg. 239

(2004) 295-303.

M.C. Dussault et al. / Forensic Science International 267 (2016) 66-72 71

[23] M. Mekel, Terrorist suicide bombings: lessons learned in Metropolitan Haifa from September 2000 to January 2006, Am. J. Disaster Med. 4 (2009) 233-248.

[24] M. Bala, N. Shussman, A.I. Rivkind, U. Izhar, G. Almogy, The pattern of thoracic trauma after suicide terrorist bombing attacks, J. Trauma Inj. Infect. Crit. Care 69 (2010) 1022-1029.

[25] I. Ashkenazi, O. Olsha, R. Alfici, K. Peleg, L. Aharonson-Daniel, M. Barham, et al., Blast injuries, N. Engl. J. Med. 352 (2005) 2651-2653.

[26] D.L. Ciraulo, E.R. Frykberg, The surgeon and acts of civilian terrorism: blast injuries, J. Am. Coll. Surg. 203 (2006) 942-950.

[27] K. Peleg, L. Aharonson-Daniel, M. Stein, M. Michaelson, Y. Kluger, D. Simon, et al., Gunshot and explosion injuries: characteristics, outcomes, and implications for care of terror-related injuries in Israel, Ann. Surg. 239 (2004) 311-318.

[28] N. Sheffy, Y. Mintz, A.I. Rivkind, S.C.A. Shapira, Terror-related injuries: a comparison of gunshot wounds versus secondary-fragments-induced injuries from explosives, J. Am. Coll. Surg. 203 (2006) 297-303.

[29] Prosecutor v Tolimir, 2010.

[30] D. Manning, Srebrenica Investigation: Summary of Forensic Evidenceexecution Point and Mass Graves, United Nations International Criminal

Tribunal for the Former Yugoslavia, The Hague, 2000.

[31] Scientific Working Group for Forensic Anthropology, Trauma Analysis, 2011. http://swganth.startlogic.com/Trauma\%20Rev0.pdf (accessed 17.07.15).

[32] J.E. Buikstra, D.H. Ubelaker, Standards for Data Collection from Human Skeletal 
Remains, Arkansas Archaeological Survey, Fayetville, 1997.

[33] B.P. Wheatley, Perimortem or postmortem bone fractures? An experimental study of fracture patterns in deer femora, J. Forensic Sci. 53 (2008) 69-72.

[34] S.A. Symes, E.N. L'Abbé, E.N. Chapman, I. Wolff, D.C. Dirkmaat, Interpreting traumatic injury to bone in medicolegal investigations, A Companion to Forensic Anthropology, Wiley-Blackwell, 2012 p. 752.

[35] S.A. Symes, E.N. L'Abbé, K.E. Stull, M. LaCroix, J.T. Pokines, Taphonomy and the timing of bone fractures in trauma analysis, Manual of Forensic Taphonomy, CRC Press, 2013, pp. 496.

[36] P. Villa, E. Mahieu, Breakage patterns of human long bones, J. Hum. Evol. 21 (1991) 27-48.

[37] D.J. Clark, Report of the Chief Pathologist-Srebrenica Related Grave Sites (With Incorporation of Findings from 1999 and 2000), 2001.

[38] R. Navarro Suay, A.H. Abadía de Barbará, C. Gutierrez Ortega, E. Bartolomé Cela, D.M. Lam, F. Gilsanz Rodríguez, Gunshot and improvised explosive casualties: a report from the Spanish Role 2 medical facility in Herat, Afghanistan, Mil. Med. 177 (2012) 326-332.

[39] K.D. Boffard, C. MacFarlane, Urban bomb blast injuries: patterns of injury and treatment, Surg. Annu. 25 (1993) 29-47.

[40] G. Almogy, Y. Mintz, G. Zamir, T. Bdolah-Abram, R. Elazary, L. Dotan, et al., Suicide bombing attacks: can external signs predict internal injuries? Ann.

Surg. 243 (2006) 541-546.

[41] M. Torkki, V. Koljonen, K. Sillanpää, E. Tukiainen, S. Pyörälä, E. Kemppainen, et al., Triage in a bomb disaster with 166 casualties, Eur. J. Trauma 32 (2006)

374-380.

[42] S.S. Hare, I. Goddard, P. Ward, A. Naraghi, E.A. Dick, The radiological management of bomb blast injury, Clin. Radiol. 62 (2007) 1-9.

[43] S. Mallonee, S. Shariat, G. Stennies, R. Waxweiler, D. Hogan, F. Jordan, Physical injuries and fatalities resulting from the Oklahoma city bombing, JAMA 276 (1996) 382-387.

[44] J.P.G. de Ceballos, Casualties treated at the closest hospital in the Madrid, March 11, terrorist bombings, Criti. Care Med. 33 (2005) S107-S112.

[45] D. Schwartz, I. Ostfeld, Y. Bar-Dayan, A single, improvised 'Kassam' rocket explosion can cause a mass casualty incident: a potential threat for future international terrorism? J. Emerg. Med. 26 (2009) 293-298.

[46] J.P. Baraybar, Variation of gunshot injury patterns in mortality associated with human rights abuses and armed conflict: an exploratory study, Sci. Justice 55

(5) (2015) 355-362.

[47] A. Petaros, M. Slaus, M. Coklo, I. Sosa, M. Cengija, A. Bosnar, Retrospective analysis of free-fall fractures with regard to height and cause of fall, Forensic Sci. Int. 226 (2013) 290-295.

[48] M. Martí, M. Parrón, F. Baudraxler, A. Royo, N.G. León, R. Álvarez-Sala, Blast injuries from Madrid terrorist bombing attacks on March 11, 2004, Emerg.

Radiol. 13 (2006) 113-122.

[49] J.P. de Ceballos, F. Turegano-Fuentes, D. Perez-Diaz, M. Sanz-Sanchez, C. Martin-Llorente, J.E. Guerrero-Sanz, 11 March 2004: The terrorist bomb explosions in Madrid, Spain-an analysis of the logistics, injuries sustained and clinical management of casualties treated at the closest hospital, Crit. Care 9 (2005) 104-111.

72 M.C. Dussault et al. / Forensic Science International 267 (2016) 66- 